\title{
THERMALIZATION \\ OF ONE-DIMENSIONAL ELECTRON GAS BY MANY-BODY COULOMB SCATTERING
}

\author{
M. MošKo AND V. CAMBeL \\ Institute of Electrical Engineering, Slovak Academy of Sciences \\ Dúbravská cesta 9, 84239 Bratislava, Slovakia
}

\begin{abstract}
Quantum wires are peculiar in the sense that binary electron-electron collisions cannot thermalize energy distribution of the electrons in the same subband. We show that such thermalization occurs through many-body Coulomb scattering. We consider one-dimensional electron gas described by Newton equations of motion with many-body Coulomb forces. These equations are solved by molecular dynamics technique. Thermalization of the non-equilibrium distribution towards Maxwell function is demonstrated for a single-subband $\mathrm{GaAs}$ wire.
\end{abstract}

PACS numbers: $73.50 .-h, 73.50 . B k, 78.47 .+p$

Optical measurements [1, 2] and Monte Carlo simulations [3, 4] of the one-dimensional (1D) carrier relaxation in GaAs quantum wires show that $1 \mathrm{D}$ carrier relaxation is much slower than in bulk and two-dimensional systems under similar conditions. Moreover, intrasubband binary collisions do not thermalize energy distribution of $1 \mathrm{D}$ electron gas at all $[3,4]$. In the intrasubband binary collision an electron changes its momentum from $k$ to $k^{\prime}$ by collision with another electron which is scattered from $k_{0}$ to $k_{0}^{\prime}$. Conservation of momentum and energy can be expressed as

$$
k+k_{0}=k^{\prime}+k_{0}^{\prime}, \quad \varepsilon(k)+\varepsilon\left(k_{0}\right)=\varepsilon\left(k^{\prime}\right)+\varepsilon\left(k_{0}^{\prime}\right) .
$$

For $\varepsilon(k)=\hbar^{2} k^{2} / 2 m$, where $m$ is the electron effective mass, one gets $k^{\prime}=k_{0}$ and $k_{0}^{\prime}=k$. Since new momentum states cannot be created, intrasubband binary collisions cannot thermalize the energy distribution. However, is such thermalization prohibited also in the $1 D$ gas with many-body Coulomb interaction? This is a fundamental question because the binary collision model is only a "Fermi's golden rule based" approximation [3-6] for the many-body problem. This paper shows that intrasubband many-body Coulomb collisions thermalize the non-equilibrium distribution of $1 \mathrm{D}$ electrons towards Maxwell distribution. Using molecular dynamics (MD), we simulate the thermalization in the GaAs wire. 
In our model the perfectly 1D electron gas with free motion in the $x$ direction occupies the lowest energy subband. The dynamics of an $i$-th electron is governed by Newton equations $\dot{k}_{i}=F_{i} / \hbar, \dot{x}_{i}=\hbar k_{i} / m$, where

$$
F_{i}=\frac{e^{2}}{4 \pi \varepsilon} \sum_{j} \frac{\left(x_{i}-x_{j}\right)}{\left|x_{i}-x_{j}\right|^{3}} .
$$

Here $F_{i}$ is the Coulomb force due to all other electrons and $\varepsilon$ is the material permittivity. (This classical MD approach is valid in the non-degenerate limit of low carrier densities $[7,8]$.) Newton equations are integrated as

$$
k_{i}(t+\Delta t)=k_{i}(t)+\Delta t \frac{F_{i}(t)}{\hbar}, \quad x_{i}(t+\Delta t)=x_{i}(t)+\Delta t \frac{\hbar k_{i}(t+\Delta t)}{m} .
$$

To deal with a finite number of particles, the following periodic boundary conditions are employed. We define a basic cell of the length $L$, which contains $N$ electrons (typically $N=1000$ ). When an electron leaves the cell crossing the boundary $x=L$, another electron is injected into the cell at the equivalent boundary $x=0$ with the same $k$ value. A similar procedure is used when an electron crosses the boundary $x=0$. Coulomb force (2) is recalculated after each time step $\Delta t$ as follows. An electron is considered to interact with $N-1$ electrons in the cell $\langle 0, L\rangle$ through specially defined interelectron distances. The calculated value of $x_{i}-x_{j}$ is used in the sum (2) only if $\left|x_{i}-x_{j}\right| \leq L / 2$. Otherwise the following replacement is used in (2). If $x_{i}-x_{j}>L / 2$, then $x_{i}-x_{j}$ is replaced by $x_{i}-x_{j}-L$. If $x_{i}-x_{j}<-L / 2$, then $x_{i}-x_{j}$ is replaced by $x_{i}-x_{j}+L$. Thus the particle at which the force is calculated sits at the center of its own cell. Since the approach is time consuming, we have also implemented a faster technique that truncates the Coulomb interaction for a pair of electrons with a distance greater than a cut-off length $R_{\mathrm{C}}<L / 2$. In this technique the sum (2) is modified using the same replacement for $\left|x_{i}-x_{j}\right|>L / 2$ as described above, however, only terms with $\left|x_{i}-x_{j}\right|<R_{\mathrm{C}}$ and terms with $\left|x_{i}-x_{j} \mp L\right|<R_{\mathrm{C}}$ are taken into account in the summation. The fast search for all electrons $j$ within the distance $R_{\mathrm{C}}$ is performed as in Ref. [9]. Above a certain $R_{\mathrm{C}}$ value we find the same results as for $R_{\mathrm{C}}=L / 2$ (no truncation).

Simulation parameters are $N=1000, \Delta t=0.1$ fs and $2 R_{\mathrm{C}}=20 / n_{L}$, where the linear electron density $n_{L}=3 \times 10^{5} \mathrm{~cm}^{-1}$ (each electron typically interacts with 20 nearest partners). At the beginning of the simulation the initial $k$ and $x$ values are selected. The size of $k$ is selected for each electron according to the Gaussian energy distribution of width $8.7 \mathrm{me}$, centered at $8.7 \mathrm{meV}$. The sign of $k$ is selected at random. Then $x$ is selected at random in the cell $\langle 0, L\rangle$ with a supplementing condition that the generation of two electrons closer than $7 \mathrm{~nm}$ is prohibited. This condition prevents initial heating and (mean kinetic energy) $/ k_{\mathrm{B}}=100 \mathrm{~K}$ during the simulation.

In Fig. 1 the occupation number versus energy and radial distribution* versus normalized distance are shown for times $0 \mathrm{fs}, 40 \mathrm{fs}, \ldots, 3$ ps after the initialization. The initial occupation number is strongly peaked as a result of the $k$ selection

*Radial distribution is the probability (per unit distance) to find a particle at the distance $d$ from another particle positioned at $d=0$. 

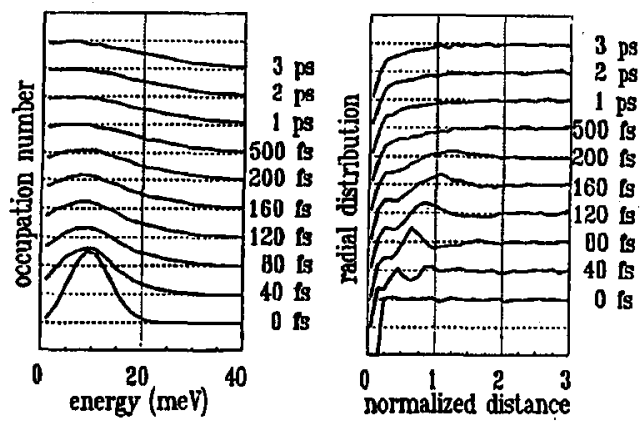

Fig. 1. Occupation number versus energy and radial distribution versus normalized distance $d / n_{L}^{-1}$ at times $0 \mathrm{fs}, 40 \mathrm{fs}, \ldots, 3 \mathrm{ps}$ after the initialization. The spacing between horizontal grid lines is 0.25 and 0.5 , respectively.

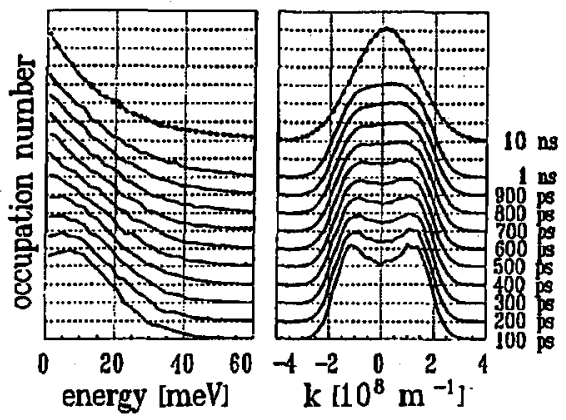

Fig. 2. Occupation number versus energy and $k$ at times $100 \mathrm{ps}, 200 \mathrm{ps}, \ldots, 10 \mathrm{~ns}$ after the initialization. The occupation number converges towards equilibrium Maxwell function at temperature $200 \mathrm{~K}$, shown in a dotted line. The spacing between horizontal grid lines is 0.05 .

from the Gaussian energy distribution. During the first picosecond one sees a fast relaxation of the occupation number towards the energy dependence which is still strongly non-Maxwellian. No remarkable change of this energy dependence is observed at times $1 \mathrm{ps}, 2 \mathrm{ps}$ and $3 \mathrm{ps}$. Radial distribution relaxes on subpicosecond time scale and remains unchanged at times $>500 \mathrm{fs}$. All the curves are averages from thirty simulations with different ordering of random numbers used to initialize $k$ and $x$.

Figure 2 shows how the occupation number from Fig. 1 progresses to relax at times $100 \mathrm{ps}, 200 \mathrm{ps}, \ldots, 10$ ns. Figure 2 also shows the corresponding dependence on $k$. The occupation number converges towards equilibrium Maxwell function, shown in a dotted line for temperature $200 \mathrm{~K}$. Curves in Fig. 2 are the time averages over 50 -ps time intervals centered at shown times.

On the nanosecond time scale of Fig. 2 the radial distribution (not shown) keeps the same shape as in Fig. 1 at times $>500$ fs. In other words, the slow thermalization in Fig. 2 occurs in the regime of equilibrium radial distribution. 
The fast relaxation of the occupation number in Fig. 1 is closely related to the relaxation of the radial distribution. The increase in the radial distribution at distances close to zero is accompanied by the increase in the occupation number at energies close to zero. This is due to the fact that at $t=0 \mathrm{a}$ part of electrons can have sufficiently high kinetic energies to move fast to very small mutual distances, where their motion is stopped by the increased repulsion. On the other hand, the increase in the high energy tail of the occupation number is due to the fact that at $t=0$ there are some close partners with high kinetic energies and oppositely orientated momenta. Repulsion of such partners further enhances their kinetic energies, thus creating the high energy tail. Generally speaking, subpicosecond relaxation in Fig. 1 takes place because the initial momenta and initial positions are uncorrelated and the system searches for a stable correlation. For example, when two electrons become much closer than the mean interparticle distance, their kinetic energies should be typically much lower than the mean kinetic energy. Even this simple correlation is disregarded at $t=0$.

Spatio-temporal oscillations of the radial distribution in Fig. 1 at times 40-200 fs show that an electron efficiently affects the dynamics of several neighbors (and vice versa). The equilibrium radial distribution within the nearest neighbor distance is smaller than the transient radial distribution, i.e. close proximity ("collision") of several electrons is less probable. This provides qualitative understanding for the slow thermalization in Fig. 2, because it occurs in the regime of equilibrium radial distribution.

We conclude that internal thermalization of the $1 D$ electron gas in a single subband occurs through many-body Coulomb scattering. Such thermalization is not predicted by Boltzmann's H-theorem. H-theorem predicts the thermalization in a gas subjected to binary collisions but there is no thermalizing effect from such collisions in the $1 \mathrm{D}$ subband.

\section{References}

[1] R. Cingolani, H. Lage, L. Tapfer, H. Kalt, D. Heitmann, K. Ploog, Phys. Rev. Lett. 67, 891 (1991).

[2] J. Christen, M. Grundmann, E. Kapon, E. Colas, D.M. Hwang, D. Bimberg, Appl. Phys. Lett. 61, 67 (1992).

[3] L. Rota, F. Rossi, S.M. Goodnick, P. Lugli, E. Molinari, W. Porod, Phys. Rev. B 47, 1632 (1993).

[4] I. Vurgaftman, J. Singh, Appl. Phys. Lett. 62, 2251 (1993).

[5] A. Mošková, M. Moško, Phys. Rev. B 49, 7443 (1994).

[6] M. Moško, A. Mošková, Semicond. Sci. Technol. 9, 478 (1994).

[7] V. Cambel, M. Moško, Semicond. Sci. Technol. 9, 474 (1994).

[8] R.P. Joshi, A.M. Kriman, M.J. Kann, D.K. Ferry, Appl. Phys. Lett. 58, 2369 (1991).

[9] V.Cambel, M. Moško, Semicond. Sci. Technol. 8, 364 (1993). 